\title{
Gamma-ray emitting narrow-line Seyfert 1 galaxies. New discoveries and open questions
}

\section{F. D'Ammando ${ }^{1} \dagger$, M. Orienti ${ }^{1}$, J. Finke ${ }^{2}$, J. Larsson ${ }^{3}$, M. Giroletti ${ }^{1}$, on behalf of the Fermi Large Area Telescope Collaboration}

\author{
${ }^{1}$ INAF - Istituto di Radioastronomia, Via Gobetti 101, I-40129 Bologna, Italy \\ ${ }^{2}$ U.S. Naval Research Laboratory, 4555 Overlook Ave. SW, Washington, DC 20375-5352, USA \\ ${ }^{3}$ KTH, Dep. of Physics, and the Oskar Klein Centre, AlbaNova, SE-106 91 Stockholm, Sweden
}

\begin{abstract}
The discovery of $\gamma$-ray emission from 5 radio-loud narrow-line Seyfert 1 galaxies revealed the presence of a possible emerging third class of AGNs with relativistic jets, in addition to blazars and radio galaxies. The existence of relativistic jets also in this subclass of Seyfert galaxies opened an unexplored research space for our knowledge of the radio-loud AGNs. Here, we discuss the radio-to- $\gamma$-rays properties of the $\gamma$-ray emitting narrow-line Seyfert 1 galaxies, also in comparison with the blazar scenario.
\end{abstract}

Keywords. galaxies: active, galaxies: nuclei, galaxies: Seyfert, gamma-rays: general

\section{Introduction}

Before the launch of the Fermi satellite only two classes of AGNs were known to produce relativistic jets and thus to emit up to the $\gamma$-ray energy range: blazars and radio galaxies, both hosted in giant elliptical galaxies (Blandford \& Rees 1978). The first 5 years of observation by the Large Area Telescope (LAT) on board Fermi confirmed that the extragalactic $\gamma$-ray sky is dominated by radio-loud AGNs, being mostly blazars and some radio galaxies. However, the discovery by Fermi-LAT of variable $\gamma$-ray emission from a few radio-loud narrow-line Seyfert 1s (NLSy1s) revealed the presence of a possible third class of AGNs with relativistic jets (Abdo et al. 2009). On the contrary, no radio-quiet Seyfert galaxies were detected in $\gamma$ rays so far (Ackermann et al. 2012).

NLSy1 is a class of AGN identified by Osterbrock \& Pogge (1985) and characterized by their optical properties: narrow permitted lines (FWHM $\left.(\mathrm{H} \beta)<2000 \mathrm{~km} \mathrm{~s}^{-1}\right)$ emitted from the broad line region (BLR), $[\mathrm{OIII}] / \mathrm{H} \beta<3$, and a bump due to Fe II (see e.g. Pogge 2000, for a review). They also exhibit strong X-ray variability, steep X-ray spectra, and substantial soft X-ray excess (e.g. Grupe et al. 2010). These characteristics point to systems with smaller masses of the central black hole $\left(\mathrm{M}_{B H}=10^{6}-10^{8} \mathrm{M}_{\odot}\right)$ and higher accretion rates (close to or above the Eddington limit) with respect to blazars and radio galaxies. NLSy1s are generally radio-quiet (radio-loudness $R<10$ ), with only a small fraction of them $(<7 \%$; Komossa et al. 2006) classified as radio-loud, and objects with high values of radio-loudness $(R>100)$ are even more sparse $(\sim 2.5 \%)$, while generally $\sim 15 \%$ of quasars are radio-loud. Considering also that NLSy1s are thought to be hosted in spiral galaxies, their detection in $\gamma$ rays poses intriguing questions about the nature of these sources, the production of relativistic jets, the mechanisms of high-energy emission, and the cosmological evolution of radio-loud AGN.

† dammando@ira.inaf.it 


\section{Radio-to- $\gamma$-ray properties of the $\gamma$-ray NLSy1s}

So far five radio-loud NLSy1 galaxies have been detected at high significance by FermiLAT: 1 H $0323+342$, SBS $0846+513$, PMN J0948 + 0022, PKS $1502+036$, and PKS 2004-447 (Nolan et al. 2012, D'Ammando et al. 2012), with a redshift between 0.061 and 0.585. Their average apparent isotropic luminosity in the $0.1-100 \mathrm{GeV}$ energy band is between $10^{44} \mathrm{erg} \mathrm{s}^{-1}$ and $10^{47} \mathrm{erg} \mathrm{s}^{-1}$, a range of values typical of blazars (D'Ammando et al. 2013b). This could be an indication of a small viewing angle with respect to the jet axis and thus a high beaming factor for the $\gamma$-ray emission, similarly to blazars. In particular, SBS $0846+513$ and PMN J0948 + 0022 showed $\gamma$-ray flaring activity combined with a moderate spectral evolution (D'Ammando et al. 2012, Foschini et al. 2011), a behaviour already observed in bright FSRQs and low-synchrotron-peaked BL Lacs (Abdo et al. 2010). Several strong $\gamma$-ray flares were observed from SBS $0846+513$ and PMN J0948 + 0022, reaching at the peak an apparent isotropic $\gamma$-ray luminosity of $\sim 10^{48}$ erg $\mathrm{s}^{-1}$, comparable to that of the bright FSRQs (Foschini et al. 2011, D'Ammando et al. 2012, D'Ammando et al. 2013d). Variability and spectral properties of these two NLSy1s in $\gamma$ rays indicate a blazar-like behaviour. Recently, an intense $\gamma$-ray flaring activity was observed by LAT also from 1H $0323+342$ (Carpenter et al. 2013). This is another indication that radio-loud NLSy1s are able to host relativistic jets as powerful as those in blazars.

Differently from the steep X-ray spectra usually observed in NLSy1s, a relatively hard X-ray spectrum was detected in the Swift-XRT observations of SBS $0846+513$ (D'Ammando et al. 2012, D'Ammando et al. 2013d), PMN J0948 + 0022 (Foschini et al. 2011, D'Ammando et al. 2014), 1H 0323 + 342 (D'Ammando et al. 2013c), and PKS 1502 + 036 (D'Ammando et al. 2013a). This suggests a significant contribution of inverse Compton radiation from a relativistic jet, similar to what is found for FSRQs. The spectral modelling of the XMM-Newton data of PMN J0948 + 0022 collected on 2011 May 28-29 showed that emission from the jet likely dominates the spectrum above $\sim 2 \mathrm{keV}$, while a soft X-ray excess is evident in the low-energy part of the X-ray spectrum (D'Ammando et al. 2014).

When observed in the radio band with the high angular resolution provided by the Very Long Baseline Array (VLBA), the $\gamma$-ray emitting NLSy1s usually show a pc-scale core-jet structure. This is the case of SBS $0846+513$ (D'Ammando et al. 2012), PKS 1502 + 036 (D'Ammando et al. 2013a), and PMN J0948 + 0022 (Giroletti et al. 2011; D'Ammando et al. 2014), although in the last two sources the jet structure is faint. The high variability brightness temperature estimated in PKS $1502+036\left(T_{B}=2.5 \times 10^{13}\right.$ $\mathrm{K}$; D'Ammando et al. 2013a), in SBS $0846+513\left(T_{B}=1.1 \times 10^{14} \mathrm{~K}\right.$; D'Ammando et al. $2013 \mathrm{~d})$, and PMN J0948 + $0022\left(T_{B}=3.4 \times 10^{11} \mathrm{~K}\right.$; Giroletti et al. 2011), strongly indicates that the jet has a Lorentz factor larger than one. An indipendent proof on the presence of beaming effects is the detection of superluminal motion of $(9.3 \pm 0.6) c$ in the jet of SBS $0846+513$ (D'Ammando et al. 2013d). On the contrary, VLBA observations did not detect apparent superluminal motion at $15 \mathrm{GHz}$ for PKS $1502+036$ during 20022012 , although the radio spectral variability and the one-sided structure seem to require the presence of boosting effects in a relativistic jet (D'Ammando et al. 2013b).

A complex connection between the radio and $\gamma$-ray emission was observed for SBS $0846+513$ and PMN J0948 + 0022, where $\gamma$-ray and radio flares have not a similar behaviour, as discussed in detail in D'Ammando et al. (2013d), D'Ammando et al. (2014), and Foschini et al. (2012).

The first spectral energy distributions (SEDs) collected for the four NLSy1s detected in the first year of Fermi operation showed clear similarities with blazars: a double- 
humped shape with a first peak in the IR/optical band due to synchrotron emission, a second peak in the $\mathrm{MeV} / \mathrm{GeV}$ band likely due to inverse Compton emission, and an accretion disc component in UV for three of the four sources. The physical parameters of these NLSy1s are blazar-like, and the jet power is in the average range of blazars (Abdo et al. 2009). The SEDs of two different activity states of SBS $0846+513$, modelled by an external Compton component of seed photons from a dust torus, could be fitted by changing the electron distribution parameters as well as the magnetic field (D'Ammando et al. 2013d), consistent with the modelling of different activity states of the blazar PKS 0208-512 (Chatterjee et al. 2013). A significant shift of the synchrotron peak to higher frequencies was observed in SBS $0846+513$ during the 2012 May flaring episode, similar to FSRQs (e.g. PKS 1510-089; D'Ammando et al. 2011). Contrary to what is observed in PMN J0948 + 0022, no significant evidence of thermal emission from the accretion disc has been observed in SBS $0846+513$ (D'Ammando et al. 2013d).

\section{Radio-loudness, host galaxies, and jet formation}

The physical parameters that drive the jet formation are still under debate. An important parameter could be the $\mathrm{BH}$ mass, with only large masses allowing an efficient jet formation (see e.g. Sikora et al. 2007). Therefore one of the most surprising fact related to the discovery of $\gamma$-ray emission from radio-loud NLSy1s was the production of a relativistic jet in objects with a relatively small BH mass $\left(10^{7}-10^{8} \mathrm{M}_{\odot}\right.$; Yuan et al. 2008). However, it is worth noting that the mass estimation of these sources has large uncertainties. In particular, Marconi et al. (2008) suggested that BLR clouds are subjected to radiation pressure from the absorption of ionizing photons. Applying a correction for this effect on the virial $\mathrm{BH}$ masses, they obtained higher values with respect to previous estimates for the NLSy1s, which are objects radiating close to their Eddington limit. Recently, Calderone et al. (2013) modelling the optical/UV data of some radio-loud NLSy1s with a Shakura \& Sunyaev disc spectrum estimated BH masses higher than $10^{8} \mathrm{M}_{\odot}$. In particular, they derived a $\mathrm{BH}$ mass of $10^{9} \mathrm{M}_{\odot}$ and $2 \times 10^{8} \mathrm{M}_{\odot}$ for PMN J0948 + 0022 and PKS $1502+036$, respectively, in agreement with the typical BH mass of blazars. This may solve the problem of the minimum BH mass predicted in different scenarios of relativistic jet formation and development, but introduces a new issue. If the BH mass of these NLSy1s is $10^{8}-10^{9} \mathrm{M}_{\odot}$, how is it possible to have such a large BH mass in a spiral galaxy?

Unfortunately only very sparse observations of the host galaxy of radio-loud NLSy1s are available up to now. Among the NLSy1s detected by LAT only for the closest one, 1H 0323 +342 , the host galaxy was clearly detected. Hubble Space Telescope and Nordic Optical Telescope observations seem to reveal a one-armed galaxy morphology or a circumnuclear ring, respectively, suggesting two possibilities: the spiral arms of the host galaxy (Zhou et al. 2007) or the residual of a galaxy merging (Anton et al. 2008). Thus the possibility that the production of relativistic jets in these objects could be due to strong merger activity, unusual in disc/spiral galaxies, cannot be ruled out.

According to the "modified spin paradigm", another fundamental parameter for the efficiency of a relativistic jet production should be the BH spin, with SMBHs in elliptical galaxies having on average much larger spins than SMBHs in spiral galaxies. This is due to the fact that the spiral galaxies are characterized by multiple accretion events with random angular momentum orientation and small increments of mass, while elliptical galaxies undergo at least one major merger with large matter accretion triggering an efficient spin-up of the SMBH. The accretion rate (thus the mass) and the spin of the $\mathrm{BH}$ seem to be related to the host galaxy, leading to the hypothesis that relativistic 
jets can form only in elliptical galaxy (e.g. Marscher et al. 2009, Böttcher et al. 2002). We noted that the $\mathrm{BH}$ masses of radio-loud NLSy1s are generally larger than those in the entire sample of NLSy1s $\left(\mathrm{M}_{\mathrm{BH}} \approx(2-10) \times 10^{7} \mathrm{M}_{\odot}\right.$; Komossa et al. 2006, Yuan et al. 2008), even if still small if compared to radio-loud quasars. The larger BH masses of radio-loud NLSy1s with respect to radio-quiet NLSy1s could be related to prolonged accretion episodes that can spin-up the BHs. In this context, the small fraction of radioloud NLSy1s with respect to radio-loud quasars could be an indication that not in all of the former the high-accretion regime lasted long enough to spin-up the central $\mathrm{BH}$ (Sikora 2009).

\section{Acknowledgement}

The Fermi LAT Collaboration acknowledges support from a number of agencies and institutes for both the development and the operation of the LAT as well as scientific data analysis. These include NASA and DOE in the United States, CEA/Irfu and IN2P3/CNRS in France, ASI and INFN in Italy, MEXT, KEK, and JAXA in Japan, and the K. A. Wallenberg Foundation, the Swedish Research Council and the National Space Board in Sweden. Additional support from INAF in Italy and CNES in France for science analysis during the operations phase is also gratefully acknowledged. FD, MO, MG acknowledge financial contribution from grant PRIN-INAF-2011.

\section{References}

Abdo, A. A., Ackermann, M., Ajello, M., et al. 2009, ApJ, 707, L142

Abdo, A. A., Ackermann, M., Ajello, M., et al. 2010, ApJ, 710, 1271

Ackermann, M., Ajello, M., Allafort, A., et al. 2012, ApJ, 747, 104

Anton, S., Browne, I. W. A., \& Marcha, M. J. 2008, A\&A, 490, 583

Böttcher, M. \& Dermer, C. D. 2002, ApJ, 564, 86

Blandford, R. D. \& Rees, M. J. 1978, in BL Lac Objects ed. A. M. Wolfe, 328

Calderone, G., Ghisellini, G., Colpi, M., \& Dotti, M. 2013, MNRAS, 431, 210

Carpenter, B. \& Ojha, R. 2013, The Astronomer's Telegram, 5344, 1

Chatterjee, R., Fossati, G., Urry, C. M., et al. 2013, ApJ, 763, L11

D'Ammando, F., Raiteri, C. M., Villata, M., et al. 2011, A\&A A, 529, 145

D'Ammando, F., Orienti, M., Finke, J., et al. 2012, MNRAS, 426, 317

D'Ammando, F., Orienti, M., Doi, A., et al. 2013a, MNRAS, 433, 952

D'Ammando, F., Tosti, G., Orienti, M., \& Finke, J. 2013b, 2012 Fermi Symposium proceedings

D'Ammando, F., Carpenter, B., \& Ojha, R. 2013c, The Astronomer's Telegram, 5352, 1

D'Ammando, F., Larsson, J., Orienti, M., et al. 2014, MNRAS, 438, 3521

D'Ammando, F., Orienti, M., Finke, J., et al. 2013d, MNRAS, 436, 191

Foschini, L., Ghisellini, G., Kovalev, Y. Y., et al. 2011, MNRAS, 413, 1671

Foschini, L., Angelakis, E., Fuhrmann, L., et al. 2012, A\&SA, 548, A106

Giroletti, M., Paragi, Z., Bignall, H., et al. 2011, A\&SA, 528, L11

Grupe, D., Komossa, S., Leighly, K. M., \& Page, K. L. 2010, ApJS, 187, 64

Komossa, S., Voges, W., Xu, D., et al. 2006, AJ, 132, 531

Marconi, A., Axon, D. J., Maiolino, R., et al. 2008, ApJ, 678, 693

Marscher, A. 2009, in Lecture Notes in Physics 794, ed. T. Belloni, 173

Nolan, P., Abdo, A. A., Ackermann, M., et al. 2012, ApJS, 199, 31

Osterbrock, D. E. \& Pogge, R. W. 1985, ApJ, 297, 166

Pogge, R. W. 2000, New Astron. Revs, 44, 381

Sikora, M., Stawarz, L., \& Lasota, J.-P. 2007, ApJ, 658, 815

Sikora, M. 2009, AN, 330, 291

Yuan, W., Zhou, H.-Y., Komossa, S., et al. 2008, ApJ, 685, 801

Zhou, H.-Y., Wang, T.-G., Yuan, W., et al. 2006, ApJS, 166, 128 